Copyright (@ 2009 Institute of Electrical and electronics Engineers, Inc.

All Rights reserved.

Personal use of this material, including one hard copy reproduction, is permitted.

Permission to reprint, republish and/or distribute this material in whole or in part for any other purposes must be obtained from the IEEE.

For information on obtaining permission, send an e-mail message to stds-igr@ieee.org.

By choosing to view this document, you agree to all provisions of the copyright laws protecting it.

Individual documents posted on this site may carry slightly different copyright restrictions.

For specific document information, check the copyright notice at the beginning of each document. 


\section{Grasping Force Estimation Detecting Slip by Tactile Sensor Adopting Machine Learning Techniques}

\author{
Abdul Md Mazid \\ Faculty of Sciences, Engineering \& Health \\ Central Queensland University \\ Rockhampton, Australia \\ Email: a.mazid@cqu.edu.au
}

\author{
A B M Shawkat Ali \\ School of Computing Sciences \\ Central Queensland University \\ Rockhampton, Australia \\ E-mail: s.ali@cqu.edu.au
}

\begin{abstract}
Adequate grasping force estimation and slip detection is a vital problem in wider applications of robots and manipulators in industries as well as in our everyday life. In this paper, a new methodology for slip detection during grasping by robot grippers/end-effectors using tactile sensor has been presented. During the object slippage, the tactile sensor in touch with the object surface travels along the peaks and valleys of surface texture of the object which creates vibratory motions in the tactile. A newly developed mathematical model is used to compute the scattered energy of vibrations, which contains parameters of surface texture geometry as well as trial grasping force, and other relevant parameters. Using the scattered energy of vibrations predicted by soft computing techniques, an attempt to instantly estimate the adequate grasping force has been reasonably successful. Surface texture data, for experimental estimation of grasping force, were collected from a huge number of machined specimens and were used to build four different machine learning estimation techniques. Experimental results using Linear Regression (LR), Simple Linear Regression (SLR), Pace Regression (PR) and Support Vector Machine (SVM) demonstrate a relatively better technique for industrial applications.
\end{abstract}

Keywords-tactile sensor, surface roughness, intelligent grasping, slip detection, support vector machine.

\section{INTRODUCTION}

Recognizing unknown objects and grasping, lifting and manipulating objects by robotic grippers / end-effectors and manipulators are crucial problems in the field of industrial applications of robots and manipulators. This covers a wide range of applications starting from diversified materials handling, such as assembly operations, peg-in-hole tasks, welding processes, grinding processes, and so on, in manufacturing sectors up to fruit and vegetable harvesting, collecting eggs in poultry farms and food processing. Another evolving problem area is that within a few decades, many industrialized countries will face a significant increase of elderly people [1]. In household tasks a robot could be used to perform simple fetch and manipulation tasks.

It is essential to assure that an object has been grasped using appropriate grasping force/s that does not allow the object slip or drop down, at the same time the gripper mechanism does not smash the grasped object or damage its surface qualities. Therefore, in grasping activities it is an essential task to detect slip or incipient slip at its very beginning stage. In this paper, an attempt has been made to detect slip of a grasped object using a recently proposed tactile sensor [2], and to estimate appropriate minimum grasping force/s from the tactile data using sophisticated machine learning techniques. The newly developed mathematical model for the scattered energy of vibrations [3] is used in conjunction with machine learning techniques to estimate the minimum appropriate grasping force for a particular object. The results of computational experiments show that machine learning techniques are efficient to estimate the grasping force.

\section{SLIP DETECTION AND FORCE ESTIMATION}

Although slip detection and grasping force estimation are supplement to each other, a few numbers of works are dedicated to their combined purpose.

Tremblay and Cutkosky (1993) presented [4] an improved dynamic tactile sensor to detect incipient slip of grasped object, which used accelerometers. By detecting a small vibration associated with incipient slip by accelerometers, a manipulator can increase its grasping force in time to prevent object slippage in robotic grasping. Another similar purpose sensor called nephelometric tactile sensor was designed, constructed, and tested by Russell [5] in 1997. The sensor was built of arrays of light-emitting diodes (LEDs) focusing on a layer of turbid liquid arrays of phototransistors. The diodes were mounted into the holes of flexible rubber block which is supposed to be distorted while in touch of object under tangential and longitudinal forces. In 2001, a similar principle of strain gauge application but simpler in design than the one used by Tsujiuchi, has been used by Yamada, Maeno and Yamada to design artificial finger skin having human like ridges on it. The authors proposed [6] to use the artificial finger skin with ridges and distributed sensor accommodated underneath the skin ridges to detect slippage during robotic grasping assessing tangential and longitudinal forces of grasp. Another tactile sensor based on strain gauge technology has been designed and published in 2004 by Tsujiuchi et al. [7] for the purposes of slip detection in robotic grasping applications assessing the triaxial forces generated during object slippage. In reality this is an array sensor built up with strain gauge based unit tactile sensors. Stress-strain on each of the strain gauges is processed using a bridge circuit. There were 60 strain gauges engaging 60 bridge circuits. However, the array sensor is able to detect slip during robotic grasping assessing the action forces of grasp.

To the best of our knowledge, at this stage, a force sensing resistor based sensor (FSR) system devised by Mingrino et al. [8] 
in 1994 is relatively more successful and physically applicable equipment to detect object slippage as well as to control grasping force simultaneously. The FSR is capable to sense simultaneously tangential and normal contact forces applied during grasp and vibrations induced by slip.

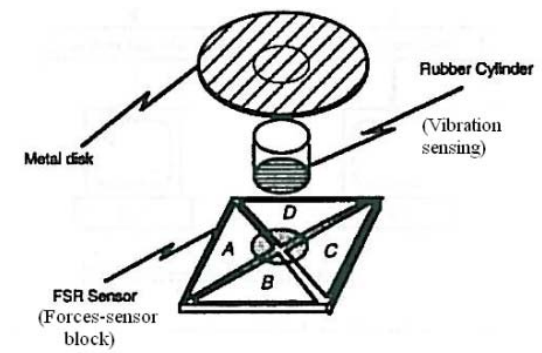

Figure 1. Exploded view of the FSR sensor system (Mingrino) [8]

The FSR sensor system, depicted in Fig. 1, consists two different sensing elements: (a) a forces-sensor block, variable resistor based on a proprietary piezoresistive semiconductor material screened on a polymer film, sensing both normal and shear forces, and (b) a vibration detector consisting of a thin metal disc mounted on a rubber cylinder which is attached to the centre of the forces-sensor block (Fig. 1). The resistance of each sensor area of the forces-sensor block provides a means to compute separately the normal and tangential components of the resultant forces acting on the metal disc in contact with the object surface. In practice the sensor system is mounted on a cylindrical tactile probe, and the probe and the sensor system are covered and secured (Fig. 2) together using an elastomeric body.

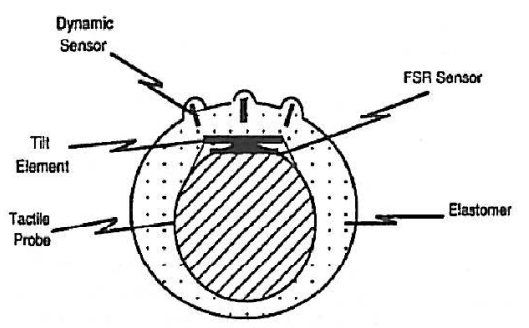

Figure 2. FSR sensor system assembled (Mingrino) [8]

\section{PHILOSOPHY OF SCATTERED ENERGY OF VIBRATIONS}

Anything in motion requires energy to keep it on motion as well as anything in motion produces 'energy' due to its motion and mass. Similarly rapidly oscillating or vibrating bodies, possessing any mass, create energy by their micro-motions. This can be explained by the fact that during the mechanical vibration of a body, the coordinate position of each components of a solid body changes in a chaotic and in some cases super-chaotic trajectory. These micro-changes of positions of a particle solid body possessing a mass clearly explain fast motion of mass through a distance. During vibrations a micro-motion of a body is always accompanied by a continuous activating force that stops activation only when vibration of the body is discontinued [3]. Therefore, if we can determine these micro translations of mass- load including their velocity, it is possible to assess the energy created by the process of chaotic oscillations of a mass. Since any amount of energy initiated by vibrations is instantly scattered away immediately after initiation in different manners, this energy is proposed [3] to call as "scattered energy of vibration" of a body.

The new parameter, called scattered energy of vibration (Esc), is philosophically the energy that is emanated during vibration [3] by the motion of a body to a particular displacement due to a particular cause and instantly it is disbursed around in the environment or in the bodies in contact.

\section{SLIP DETECTION AND SCATTERED ENERGY OF VIBRATION}

The tactile / stylus method is a recognized and widely used method to determine the grade of surface texture in manufacturing industries. If a stylus slides across a surface it goes through the peaks and valleys of waviness and roughness of the particular surface. This across-surface motion of a stylus following peaks and valleys creates reciprocating vibratory motions of the stylus itself along its axis perpendicular to the nominal surface as depicted in Figure 3.

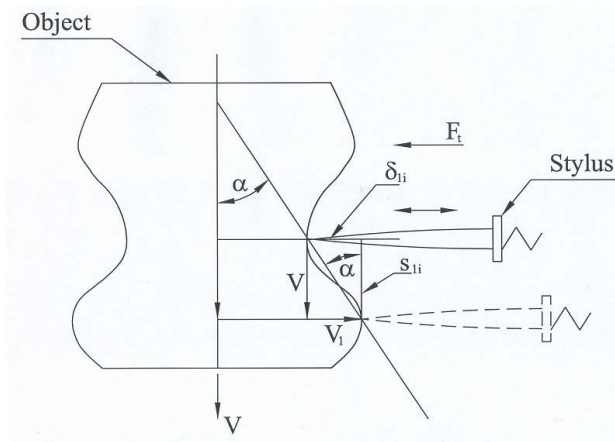

Figure 3. Sensing slippage by a stylus [2]

A tactile/stylus embedded into the gripper or fingers of a robot can sense any slippage of grasped object while the stylus slides across the peaks and valleys of surface irregularities or surface waviness. The linear reciprocating motion of a stylus can be modeled in the form of newly developed scattered energy of vibrations.

In this particular case, the Scattered Energy of Vibration (Esc) is the amount of energy emanated, as well as instantly dissipated, due to vibrations of stylus, which could be determined by a newly developed equation expressed as follows [3]:

$$
E_{s c}=n \cdot F_{t r} \cdot v \cdot \tan \sum_{i=1}^{n}\left(\cot \frac{\delta_{1 i}}{s_{1 i}}\right)
$$

where, $\mathrm{E}_{\mathrm{sc}}$ - total scattered energy of vibration; $\mathrm{F}_{\mathrm{tr}}$ - trialgrasping force possible to manipulate by a control system; $\mathrm{n}-$ number of irregularities a stylus has sensed during an object slippage; $\mathrm{v}$ - falling velocity of the slipping object; $\delta_{1 \mathrm{i}}, \mathrm{S}_{1 \mathrm{i}}$ geometry of surface irregularities of the object. Equation (1) demonstrates that, Esc is directly proportional to the number of 
peaks and valleys, their geometry sensed by the stylus during slippage, and also the falling velocity of the object.

Since it is a necessity to apply enough grasping force instantly, when the object demonstrates any incipient slip, to stop slippage, the displacement travelled by a falling body can be considered as a micro-displacement. In cases of microdisplacement, the falling velocity $\mathrm{v}$ of any body of any mass can be again considered to be the same and is a constant vector.

\section{OBJECT-SAMPLES FOR EXPERIMENT}

As mentioned objects in nature may have various surface textures with extremely different nature waviness and roughness as well as different geometry of peaks and valleys. Therefore, for industrial applications of robots, it is not possible to model object surface-textures on universal basis. But we can presume particular areas of applications of robot-systems. In this research work, authors have been concentrated on machined surfaces obtained by turning and milling processes.

In turning processes a $0.50 \mathrm{~m}$ long mild steel bar with diameter $32 \mathrm{~mm}$ was divided into 13 sections after a preliminary turn to make it of consistent diameter all through. Each section was turned separately using commonly used carbide turning bit of conventional geometry on a universal lathe machine of model Harrison M400 made by T S Harrison \& Sons Ltd., England using various surface cutting speeds starting from $\mathrm{V}=10 \mathrm{~m} / \mathrm{min}$. up-to $\mathrm{V}=161 \mathrm{~m} / \mathrm{min}$, depth of cut $\mathrm{t}=0.40 \mathrm{~mm}$, and feed rate $\mathrm{f}=$ $0.18 \mathrm{~mm} / \mathrm{rev}$. Then surface roughness values $\mathrm{Rz}$ were measured (Fig. 4) using a surface roughness measurement machine Suntronic 3+ produced by Rank Taylor Hobson Limited, England. For each section of machining, measurements were taken at three different sections approximately at an equal angular distance $\left(\sim 120^{\circ}\right)$ around the circumference. That gave us altogether about 39 different $\mathrm{Rz}$ values which have been used to represent 39 different objects for sensing purposes.

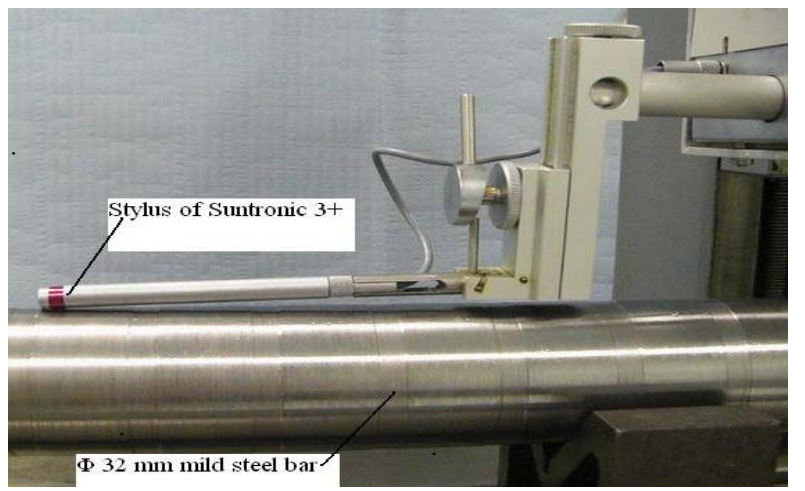

Figure 4. Measurement of surface roughness (Rz) using Suntronic 3+

Similarly another about 90 mild steel specimens were prepared by milling operations, and surface roughness values $\mathrm{Rz}$ were measured using the same Suntronic 3+ machine as depicted in Fig. 5. In milling operations depth of cut and feed rate have been kept constant and accepted as $\mathrm{t}=0.50 \mathrm{~mm}$ and $\mathrm{f}=460$ $\mathrm{mm} /$ min correspondingly.

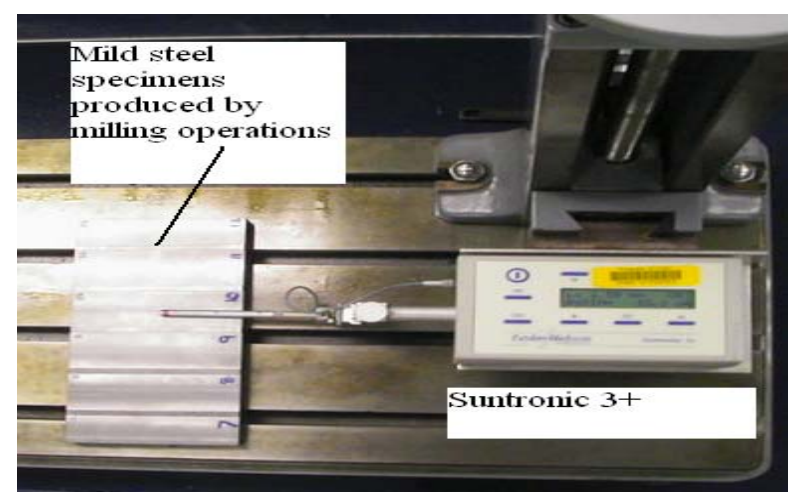

Figure 5. Surface roughness (Rz) of milled specimens using Suntronic 3+

In all cases of surface roughness measurement using Suntronic 3+, sampling length Lc $=2.50 \mathrm{~cm}$ has been accepted for more accurate values. These $\mathrm{Rz}$ values represent $\delta_{1 \mathrm{i}}$ parameter in Equation (1), and values proportionate to $\delta_{1 \mathrm{i}}$ have been accepted for the parameter $\mathrm{S}_{1 \mathrm{i}}$ for simplicity in this work. Applying these experimental parameters in Equation (1) second series of experiments has been carried out with the help of soft computing technique as described in the following sections.

\section{MACHINE LEARNING FOR GRASPING FORCE ESTIMATION}

In the second experimental stage of grasping force estimation on detecting object slip using scattered energy of vibration method, we employed four machine learning estimation techniques: Linear Regression [9], Simple Linear Regression [10], Pace Regression [11], and Support Vector Machine (SVM) to test grasping force estimation. In these experiments, we tested two types of optimisation methods for SVM: Sequential Minimal Optimization (SMOreg) [12] and advanced Sequential Minimal Optimization (SVMreg) [13].

\section{A. Support Vector Regression (SVR)}

At the beginning we consider a set of training data $\left\{\left(\mathbf{x}_{i}, y_{1}\right), \ldots,\left(\mathbf{x}_{i}, y_{i}\right)\right\}$, where $\mathbf{x}_{i}$ and $y_{i}$ represent the geometric parameters of surface roughness of grasped sample-object. Here each $\mathbf{x}_{i} \subset R^{n}$ denotes the input space of the sample and has a corresponding target value $y_{i} \subset R$ for $\mathrm{i}=1, \ldots, l$ where $l$ represents the size of the training data $[14,15]$. The idea of the regression problem is to determine a function that can estimate a target value from a given input value accurately.

As like SVM classification, the generic SVR estimating function takes the form:

$$
f(x)=\{(\mathbf{w} \cdot \Phi(\mathbf{x})\}+b
$$

where $\mathbf{w} \subset R^{n}, b \subset R$ and $\Phi$ denotes a non-linear transformation from $R^{n}$ to high dimensional space; $\mathbf{w}=$ weight vector and $b=$ bias factor. Our goal is to find the value of $\mathbf{w}$ and 
$b$ such that values of $\mathbf{x}$ can be determined [14] by minimizing the regression risk $R_{\text {reg }}(f)$ as follows:

$$
R_{\text {reg }}(f)=C \sum_{i=0}^{\ell} \Gamma\left(f\left(\mathbf{x}_{i}\right)-y_{i}\right)+\frac{1}{2}\|\mathbf{w}\|^{2}
$$

where $\Gamma(\cdot)$ is a cost function, $C$ is a constant parameter and vector $\mathbf{W}$ can be written in terms of data points [14] as:

$$
\mathbf{w}=\sum_{i=1}^{\ell}\left(\alpha_{i}-\alpha_{i}^{*}\right) \Phi\left(\mathbf{x}_{i}\right)
$$

By substituting equation (3) into equation (1), the generic equation can be rewritten as:

$$
\begin{aligned}
f(x) & =\sum_{i=1}^{\ell}\left(\alpha_{i}-\alpha_{i}^{*}\right)\left(\Phi\left(\mathbf{x}_{i}\right) \cdot \Phi(\mathbf{x})\right)+b \\
& =\sum_{i=1}^{\ell}\left(\alpha_{i}-\alpha_{i}^{*}\right) k\left(\mathbf{x}_{i}, \mathbf{x}\right)+b
\end{aligned}
$$

In equation (5), the dot product can be replaced with function $k\left(\mathbf{x}_{i}, \mathbf{x}\right)$, also called the kernel function. All kernel functions must satisfy Mercer's condition [14] that corresponds to the inner product of some feature space. The radial basis function (RBF) is commonly used as the kernel for regression and expressed as

$$
k\left(\mathbf{x}_{i}, \mathbf{x}\right)=\exp \left\{-\gamma\left|\mathbf{x}-\mathbf{x}_{j}\right|^{2}\right\}
$$

where $\gamma=$ smoothening parameter.

We have experimented with the two kernel functions: Linear and Polynomial.

The $\varepsilon$ - insensitive loss function is the most widely used cost function [15]. The function is in the form:

$$
\Gamma(f(x)-y)= \begin{cases}|f(\mathbf{x})-y|-\varepsilon, & \text { for }|f(\mathbf{x})-y| \geq \varepsilon \\ 0 & \text { otherwise }\end{cases}
$$

By solving the quadratic optimization problem in (7), the regression risk in equation (3) and the $\varepsilon$ - insensitive loss function (7) can be minimized as follows:

$$
\Gamma(f(x)-y)=\frac{1}{2} \sum_{i, j=1}^{\ell}\left(\alpha_{i}^{*}-\alpha_{i}\right)\left(\alpha_{j}^{*}-\alpha_{j}\right) k\left(\mathbf{x}_{i}, \mathbf{x}_{j}\right)-\sum_{i=1}^{\ell} \alpha_{i}^{*}\left(y_{i}-\varepsilon\right)-\alpha_{i}\left(y_{i}+\varepsilon\right)
$$

This is subjected to the fulfillment of the following conditions:

$$
\sum_{i=1}^{\ell} \alpha_{i}-\alpha_{i}^{*}=0, \quad \alpha_{i}, \alpha_{i}^{*} \in[0, C]
$$

The input vectors corresponding to the non-zero Lagrange multipliers, $\propto_{i}$ and $\propto_{i}^{*}$ are known as support vectors, which pay the significant role to construct the regression line. Only if the requirement $|f(x)-y| \geq \varepsilon$ (Figure 6) is fulfilled, Lagrange multipliers may be non-zero values and is used as support vectors.
Now, we have solved the value of $\boldsymbol{w}$ in terms of the Lagrange multipliers. For the variable $b$, it can be computed by applying Karush-Kuhn-Tucker (KKT) conditions which, in this case, implies that the product of the Lagrange multipliers and constrains leads to zero:

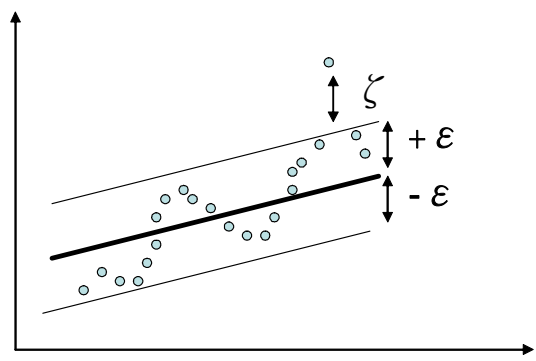

Figure 6. Support vector regression to fit a tube with radius $\varepsilon$

$$
\begin{aligned}
& \alpha_{i}\left(\varepsilon+\zeta_{i}-y_{i}+\left(w, \mathbf{x}_{i}\right)+b\right)=0 \\
& \alpha_{i}^{*}\left(\varepsilon+\zeta_{i}^{*}+y_{i}-\left(\mathbf{w}, \mathbf{x}_{i}\right)-b\right)=0
\end{aligned}
$$

and

$$
\left(C-\alpha_{i}\right) \zeta_{i}=0, \quad\left(C-\alpha_{i}^{*}\right) \zeta_{i}^{*}=0
$$

Where $\zeta_{i}$ and $\zeta_{i}^{*}$ are slack variables used to measure errors outside the $\varepsilon$-tube. Since $\alpha_{i}, \alpha_{i}^{*}=0$ and $\zeta_{i}^{*}=0$ for $\alpha_{i}^{*} \in$ $(0, C), b$ can be computed as follows:

$$
\begin{array}{ll}
b=y_{i}-\left(w, \mathbf{x}_{i}\right)-\varepsilon & \text { for } \alpha_{i} \in(0, C) \\
b=y_{i}-\left(w, \mathbf{x}_{i}\right)+\varepsilon & \text { for } \alpha_{i}{ }^{*} \in(0, C)
\end{array}
$$

Putting it all together, we can use SVM and SVR without knowing the Kernel transformation.

The rational for choosing SVM is its demonstrated improved performance in many areas including business, engineering, medical, and science $[16,17,18]$.

\section{B. Experimental Results}

We consider a set of performance metrics to evaluate the effectiveness of the estimation techniques which are listed in Table 1 with their mathematical notation [17]: The Correlation Coefficient (CC) basically compares the predicted values with actual values. If the predicted and actual values are same then the $\mathrm{CC}$ is 1 . On the other hand, if there have no correlation between the predicted and actual values then the CC is 0 . The Mean Absolute Error (MAE) and Root Mean Squared Error (RMSE) represent the basic difference between the predicted and actual values without considering more details as like CC. The Relative Absolute Error (RAE) and Root Relative Squared Error are very similar but bit details error measures approach as comparing with MAE and RMSE.

We collected data for $\delta_{1 \mathrm{i}}$ and $\mathrm{S}_{1 \mathrm{i}}$ : geometry of surface irregularities of the object from the laboratory. Then we measure the $F_{t r}$ - trial-grasping force possible to manipulate by a control system. We placed all these values in the Eqn. (1) to calculate Esc - total scattered energy of vibration. In our experiment, the estimation models were built using the collected samples of $\delta_{1 \mathrm{i}}$, 
$\mathrm{S}_{1 \mathrm{i}}$ and Esc. Once the model was built, unknown $\delta_{1 \mathrm{i}}, \mathrm{S}_{1 \mathrm{i}}$ not used in training were presented to the model to predict Esc. We considered 104 samples in our experiment and used 10 fold cross validation method as it has been suggested in $[20,21]$ for datasets having fewer than 1000 instances.

\begin{tabular}{|c|c|}
\hline TABLE & PERFORMANCE METRICS USED \\
\hline $\begin{array}{c}\text { Correlation } \\
\text { Coefficient (CC) }\end{array}$ & $C C=\frac{\frac{1}{n-1} \sum_{i}\left(Y_{i}-\bar{Y}_{i}\right)\left(Y^{*}{ }_{i}-\overline{Y_{i}^{*}}\right)}{\sigma_{Y_{i}} \sigma_{Y_{i}^{*}}}$ \\
\hline $\begin{array}{l}\text { Mean Absolute } \\
\text { Error (MAE) }\end{array}$ & MAE $=\frac{1}{n} \sum_{i=1}^{n}\left(Y_{i}-Y_{i}^{*}\right)$ \\
\hline $\begin{array}{l}\text { Root Mean } \\
\text { Squared Error } \\
\text { (RMSE) }\end{array}$ & $R M S E=\sqrt{\frac{1}{n} \sum_{i=1}^{n}\left(Y_{i}-Y_{i}^{*}\right)^{2}}$ \\
\hline $\begin{array}{l}\text { Relative Absolute } \\
\text { Error (RAE) in } \%\end{array}$ & $R A E=\left|\frac{Y_{i}-Y_{i}^{*}}{Y_{i}^{*}-\overline{Y^{*}}}\right| x 100$ \\
\hline $\begin{array}{l}\text { Root Relative } \\
\text { Squared Error } \\
\text { (RRSE) in \% }\end{array}$ & $R M S E=\sqrt{\sum_{i=1}^{n}\left(\frac{Y_{i}-Y_{i}^{*}}{Y_{i}^{*}-Y^{*}}\right)^{2} x 100}$ where $\overline{Y^{*}}=\frac{1}{n} \sum_{i}^{Y^{*}}$ \\
\hline
\end{tabular}

We used above measures to choose a superior estimation technique for grasping force estimation and presented in Figures 7-9. We tested the individual measure with each estimation technique. Then we ranked highest number of best performances to choose the best estimation technique, found SVM is the superior technique for the grasping force estimation than the others used.

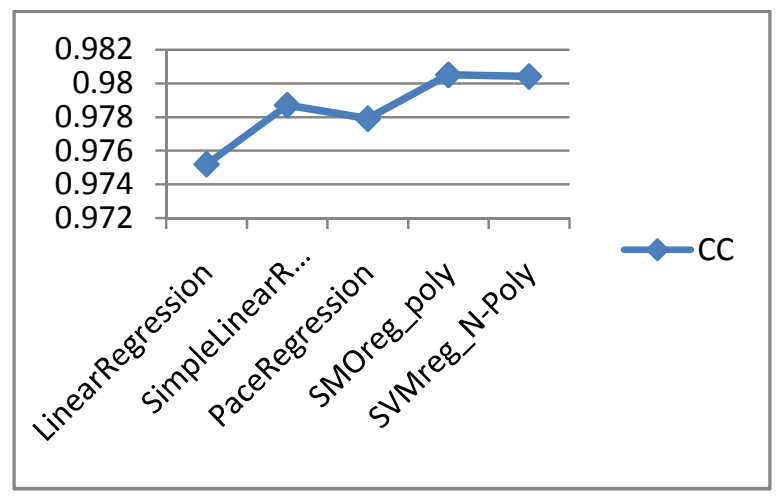

Figure 7. Grasping force estimation comparison based on CC.

Results demonstrate that SVM contineously outperformed other techniques in our experiments to predict estimated grasping forces. SVM method has option to trial different types of kernel [14]. Basically linear (lin), polynomial (poly) and radial basis function (rbf) are the classical kernel of SVM. Therefore, we investigate different types of kernel performance with two types SVM optimization techniques. We tested one more kernel called normalized polynomial (N-poly) rather than classical kernels only in our experiment. Finally the results were verified using all of the estimation error measures but reported only two of them in Figures 10-11 due to space limitation.

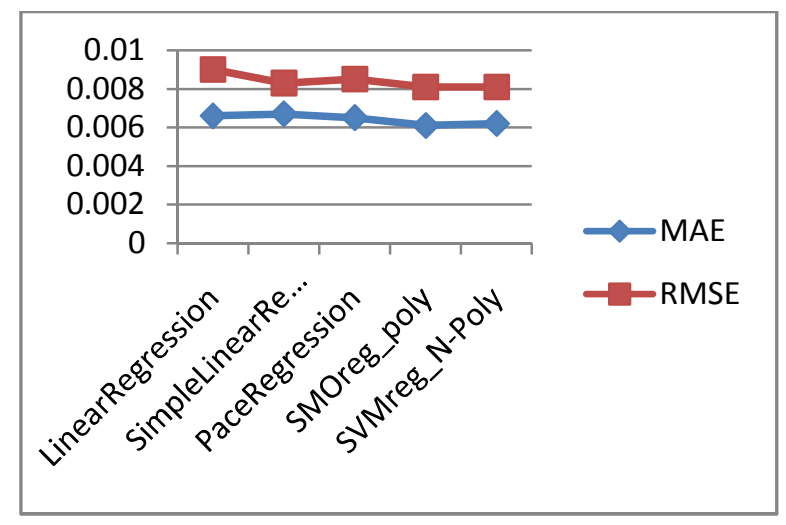

Figure 8. Grasping forces estimation based on MAE and RMSE

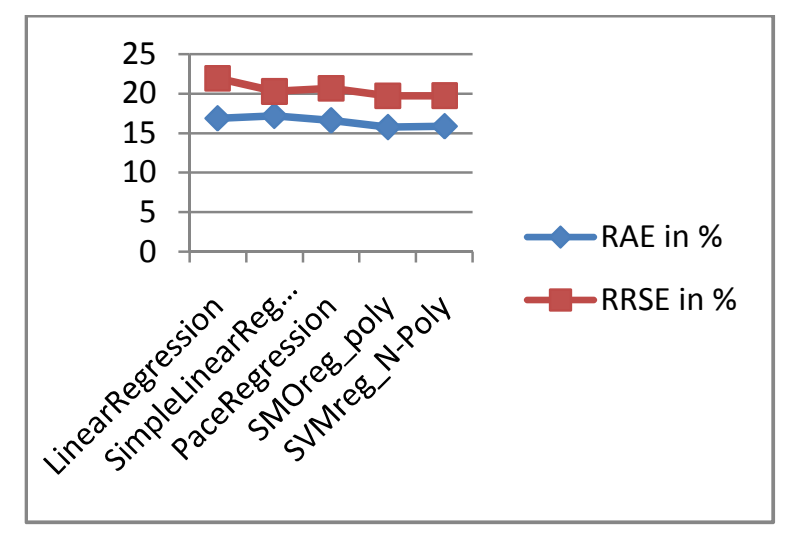

Figure 9. Grasping forces estimation based on RAE and RRSE in \%.

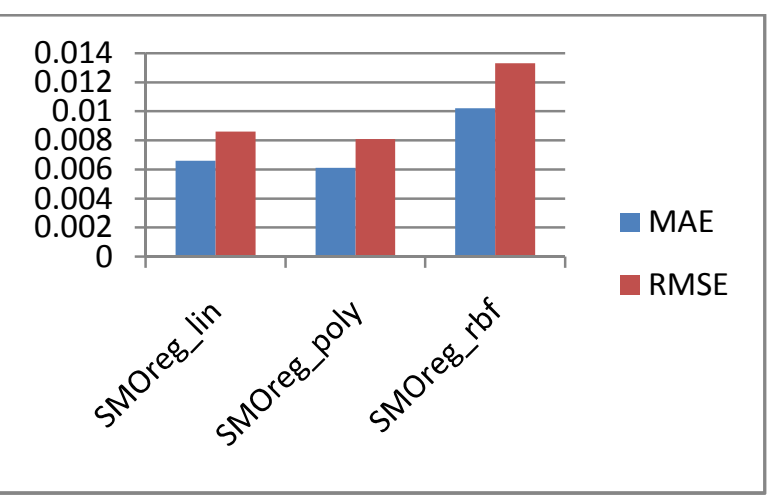

Figure 10. Comparative SVM kernel performance with SMO 


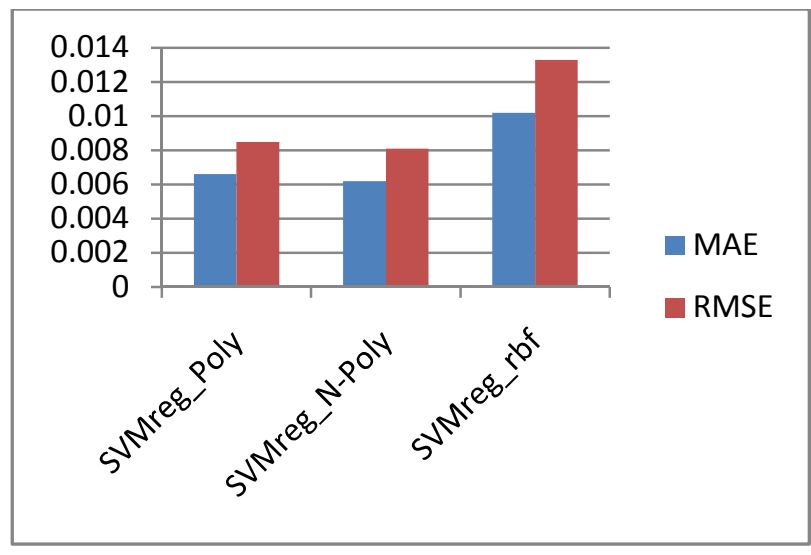

Figure 11. Comparative SVM kernel performance with SVMreg

We observed polynomial kernel is the best suited kernel for grasping force estimation. The classical polynomial kernel performed best with SMO optimization methods. We found the best suited polynomial degree was 2 . On the other hand normalized polynomial kernel performed best with SVMreg. Moreover, the performance could be improved using kernel parameter tuning.

\section{CONCLUSION}

A proposed new type of tactile sensor has been successfully applied to detect object slippage during grasping by robotic grippers/end-effectors, which allows estimating adequate grasping forces theoretcally. The newly developed mathematical model for grasping force estimation has been used to built support vector machine and other soft computing models capable of estimating grasping force from the surface irregularities of the grasped object. Computational experiments and analysis showed that the support vector based techniques estimated the grasping force with reasonably acceptable accuracy. Further improvement through the use of additional surface parameters and optimization techniques [22] to optimize SVM and kernel parameters is currently under investigation.

\section{ACKNOWLEDGEMENT}

The authors sincerely acknowledge active helps of Garry Hoar and Ian Major, Engineering Workshop, CQU Rockhampton, QLD, Australia for preparing mild steel samples for surface roughness studies by turning and milling processes in due course.

\section{REFERENCES}

[1] Johan Tegin and Jan Wikander, Tactile sensing in intelligent robotic manipulation - a review. Industrial Robot: An International Journal, v32 / No.1, 2005. Pp $64-70$.

[2] M. A. Mazid, M. Fakhrul Islam - Grasping Force Estimation Recognising Object Slippage by Tactile Data Using Neural Network. 2008 IEEE International Conference on Robotics, Automation and Mechatronics (RAM 2008). Chengdu, China. June 2008.

[3] M. A. Mazid - Scattered energy of vibrations: a novel parameter for rotating shaft vibration assessment. Proceedings, Fifth Australian Congress on Applied Mechanics 2007(ACAM 2007), Brisbane. Pp. 508-513.
[4] Marc R. Tremblay, Mark R. Cutkosky, Estimating Friction Using Incipient Slip Sensing During a Manipulation Task. Proceedings of IEEE International Conference on Robotics and Automation. Vol. 1. Atlanta, Georgia. 1993. pp. 429-434.

[5] R. Andrew Russell, A Nephelometric Tactile sensor. Advanced Topics in Artificial Intelligence - Proceedings of $10^{\text {th }}$ Australian Joint Conference on Artificial Intelligence, AI'97. Perth, Australia. 1997. pp. 467-474.

[6] Daisuke Yamada, Takashi Maeno, and Yoji Yamada, "Artificial Finger Skin having Ridges and Distributed Tactile Sensors used for Grasping Force Control". Proceedings of the 2001 IEEE/RSJ International Conference on Intelligent Robots and systems. Maui, Hawaii, USA, 2001 pp. $686-691$.

[7] Nobutaka Tsujiuchi, T. Koizumi, A. Ito, H. Oshima, Y. Nojiro, Y. Tsuchiya, and S. Kurogi, "Slip Detection with Distributed-Type Tactile Sensor. Proceedings of 2004 IEEE/RSJ International Conference on Intelligent Robotics and Systems, Sendale, Japan, 2004. pp. 331-336.

[8] Mingrino, A., Bucci, A., Magni, R., and Dario, P., "Slippage Control in Hand Prostheses by sensing Grasping Forces and Sliding Motion." IEEE International Conference on Intelligent Robots and Systems, v3, 1994, pp. 1803-1809.

[9] Daper, N.R. and Smith, H. Applied Regression Analysis .Wiley Series in Probability and Statistics, 1998.

[10] Gerard E. Dallal, Introduction to Simple Linear Regression, http://www.tufts.edu/ gdallal/slr.htm: $12^{\text {th }}$ May 2007.

[11] Yong Wang. A new approach to fitting linear models in high dimensional spaces. PhD thesis, University of Waikato, Department of Computer Science, Hamilton, New Zealand, 2000.

[12] J. Platt, Fast Training of Support Vector Machines using Sequential Minimal Optimization, in Advances in Kernel Methods - Support Vector Learning, B. Schölkopf, C. Burges, and A. Smola, eds., MIT Press, to appear, 1998.

[13] S.K. Shevade, S.S. Keerthi, C. Bhattacharyya, and K.R.K. Murthy: Improvements to the SMO Algorithm for SVM Regression. In: IEEE Transactions on Neural Networks, 1999.

[14] V.N. Vapnik. The Nature of Statistical Learning Theory. Springer, New York, 1995.

[15] K.R. Muller, A. Smola, G. Ratch, B. Scholkopf, J. Kohlmorgen and V Vapnik, "Using Support Vector Support Machines for Time Series Prediction", Image Processing Services Research Lab, AT\&T Labs.

[16] Kamruzzaman, J., Sarker, R.A. and Ahmad, I. "SVM based models for predicting foreign currency exchange rates," Proc. IEEE International Conference on Data Mining (ICDM 2003), 2003, Pp. 557- 560, 2003.

[17] Kamruzzaman, J. and Begg, R. "Support vector machines and other pattern recognition approaches to the diagnosis of cerebral palsy gait," IEEE Trans on Biomedical Engg., vol. 53, no. 12, 2006, Pp. 2479-2490.

[18] R. Begg and J. Kamruzzaman, "A machine learning approach for automated recognition of movement patterns using basic, kinetic and kinematic gait data," Journal of Biomechanics, vol. 38, pp. 401-408, 2005.

[19] I. H. Witten, and E. Frank, "Data Mining: Practical Machine Learning Tool and Technique with Java Implementation" Morgan Kaufmann, San Francisco, 2000.

[20] Henery, R. J., "Methods of Comparison" in D. Michie, et al., (eds.), Machine Learning, Neural and Statistical Classification, Ellis Horwood Limited, New York. 1994.

[21] Ali, S. and Wasimi, S., "Data Mining: Methods and Techniques." Thomson Publishers, Melbourne, 2007.

[22] Sarker, R., Kamruzzaman, J. and Newton, C. "Evolutionary Optimization (EvOpt): A Brief Review and Analysis", International Journal of Computational Intelligence and Applications, vol. 3, no. 4, 2003, Pp1-20. 